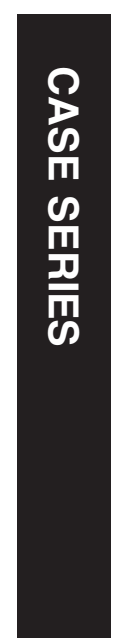

\title{
Choroidal excavation in choroidal osteoma complicated by choroidal neovascularization
}

\begin{abstract}
Purpose To describe multimodal imaging features of choroidal osteoma (CO) complicated by choroidal neovascularization (CNV) and focal choroidal excavation (FCE). Methods Patients presenting with $\mathrm{CO}$ and CNV between January and October 2016 were considered for this study. Diagnosis of CO was confirmed by ultrasound examination. All patients underwent multimodal imaging including optical coherence tomography (OCT), swept-source OCT angiography (DRI OCT Triton, Topcon, Inc., Tokyo, Japan) and fluorescein angiography (Spectralis HRA +OCT; Heidelberg Engineering, Heidelberg, Germany).

Results Two patients (one with bilateral CO) were included in the study. OCT showed a FCE in two eyes of two patients (one in correspondence of the CNV and the other adjacent to the CNV). OCT-A demonstrated presence of microvascular flow within neovascular network of the CNVs. Decalcification of the tumor was noted in correspondence of one eye with FCE. Conclusions FCE may be found in eyes with choroidal osteoma and CNV. OCT-A was a valuable tool for detection of $\mathrm{CNV}$ complicating choroidal osteoma.

Decalcification of choroidal osteoma may represent a common pathogenic pathway for development of FCE and CNV in choroidal osteoma.
\end{abstract}

Eye (2017) 31, 1740-1743; doi:10.1038/eye.2017.136; published online 21 July 2017

\section{Introduction}

Choroidal osteoma (CO) is a rare benign ossifying tumor with mature bone replacing choroid and presenting as a yellow-orange
L Pierro, A Marchese, M Gagliardi, U Introini, M Battaglia Parodi, G Casalino and F Bandello lesion on fundus examination. ${ }^{1}$ Natural course of $\mathrm{CO}$ may include tumor growth, overlying alterations of the retinal pigment epithelium (RPE), subretinal fluid, decalcification and choroidal neovascularization $(\mathrm{CNV}){ }^{2} \mathrm{CNV}$ is by far the most sight threatening complication of $\mathrm{CO}$ and may be related to tumor decalcification. ${ }^{2}$

Focal choroidal excavation (FCE) is a relatively recent optical coherence tomography (OCT) finding characterized by one or more focal areas of choroidal depression without any evidence of scleral ectasia or staphyloma. ${ }^{3,4}$ Since originally reported, ${ }^{3}$ FCE etiology is still unclear. FCE has been reported in otherwise healthy eyes ${ }^{3-5}$ or in eyes presenting with a broad spectrum of chorioretinal diseases, including eyes with $\mathrm{CNV} .{ }^{6-8}$

Aim of this study was to describe multimodal imaging features of $\mathrm{CO}$ complicated by $\mathrm{CNV}$ and FCE. We considered patients presenting with $\mathrm{CO}$ complicated by CNV between January and October 2016 at our department. Patients with any ocular conditions other than $\mathrm{CO}$ were excluded.

Diagnosis of CO was confirmed by ophthalmic standardized echography (A and B scan). When present, tumor decalcification was defined as pale areas within the osteoma showing loss of bone, RPE thinning and visibility of underlying choroidal vessels. ${ }^{2,9}$

All patients underwent multimodal imaging, including fluorescein angiography (FA) and OCT angiography (OCT-A, DRI OCT Triton, Topcon, Inc., Tokyo, Japan). Two patients were included in the study.

\section{Case 1}

A 40-year-old man presented with recent onset of metamorphopsia in the right eye (RE). He had history of bilateral $\mathrm{CO}$, complicated by a FCE in the RE and a long-standing CNV in the left eye (LE), which had been treated with three 
intravitreal injections of ranibizumab. His medical history was otherwise unremarkable. Best corrected visual acuity (BCVA) was 76 ETDRS letters (20/32 Snellen equivalent) in the RE with $-3.00 \mathrm{sph}$ and counting fingers in the LE.

OCT in the RE revealed a subretinal hyperreflective material in correspondence of the FCE. FA showed an active leaking $\mathrm{CNV}$, which was well delineated on OCT-A (Figure 1). Three monthly intravitreal injections of ranibizumab in the RE were planned. One month after the third injection BCVA was stable, FA showed no leakage and no changes of FCE were noted on OCT.

\section{Case 2}

A 29-year-old woman was referred for examination of a right long-standing $\mathrm{CO}$ complicated by $\mathrm{CNV}$ which had been treated with four intravitreal injections of ranibizumab. Clinical history was otherwise unremarkable. BCVA was 45 ETDRS letters (20/125 Snellen equivalent) with $-2.75 \mathrm{sph}$ in the RE and 83 letters (20/25 Snellen equivalent) with $-2.25 \mathrm{sph}$ in the LE.

Fundus examination in the RE showed a large CO. Around the optic disc, a pale area within the $\mathrm{CO}$ with visible underlying choroidal vessels was suggestive of decalcification of the CO. ${ }^{2,9}$ Fundus autofluorescence and OCT showed loss of RPE in the decalcified area (Figure 2). FA showed a partly leaking subfoveal CNV whose boundaries were well delineated by OCT-A. OCT in the RE showed subretinal hyperreflective material associated with subretinal fluid. In correspondence of the decalcified area, a FCE was noted (Figure 2). One intravitreal injection of ranibizumab was planned. However the patient did not attend the injection clinic and was lost to follow-up.

\section{Discussion}

In our series two eyes of two patients developed $\mathrm{CNV}$ in correspondence or in proximity of a FCE. This is not surprising as $\mathrm{CNV}$ has been reported to occur on the slope or the bottom of eyes with FCE. ${ }^{7,8}$ Margolis et al ${ }^{4}$ classified FCE in conforming lesions, in which the overlying retina is close to the retinal pigment epithelium (RPE), and nonconforming lesions in which a hyporeflective space is visible between the retina and RPE. He also hypothesized that FCE may be a congenital posterior segment malformation initially developing as a conforming lesion and subsequently progressing in a
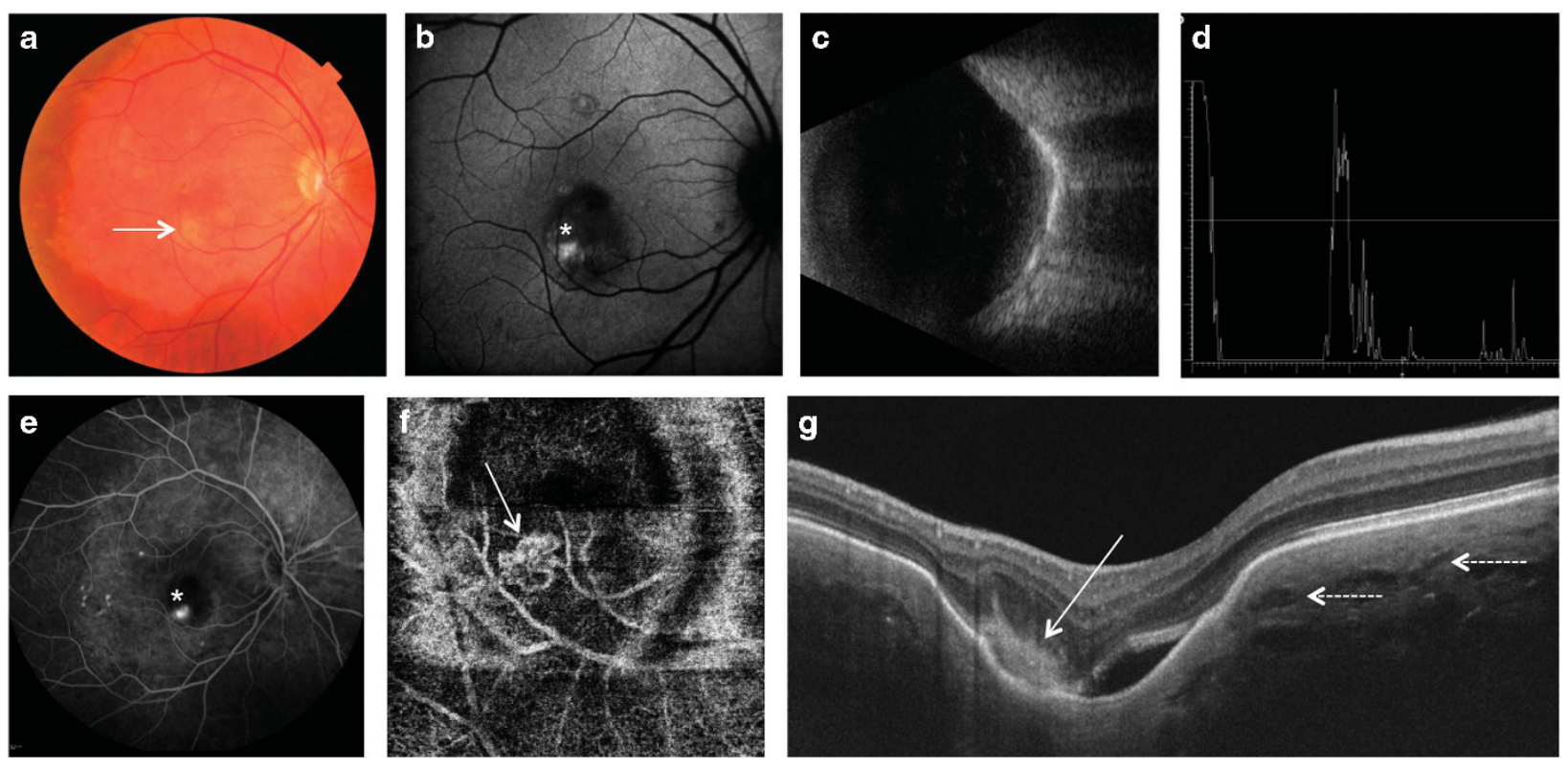

Figure 1 Multimodal imaging of right eye in case 1. Color fundus photograph (a) showed a large choroidal osteoma at the posterior pole of the right eye. A more intense yellowish material was noted in the fovea (arrow) which was hyperautofluorescent (asterisk) on blue fundus autofluorescence (b). B scan echography (c) showed a solid highly reflective choroidal mass with acoustic shadowing. A scan ultrasonography (d) showed high intensity echo spike with sound attenuation posterior to the lesion. Axial length on A scan was $24.00 \mathrm{~mm}$. Fluorescein angiography (e) showed an active leaking juxtafoveal choroidal neovascularization (CNV) which was visible on optical coherence tomography angiography (f), (arrow). Enhanced depth imaging optical coherence tomography (g) showed a choroidal sponge-like pattern with the presence of multiple intralesional layers (dashed arrows); in correspondence of the CNV an ill defined hyperreflective material was found on the slope of the choroidal excavation (arrow). 

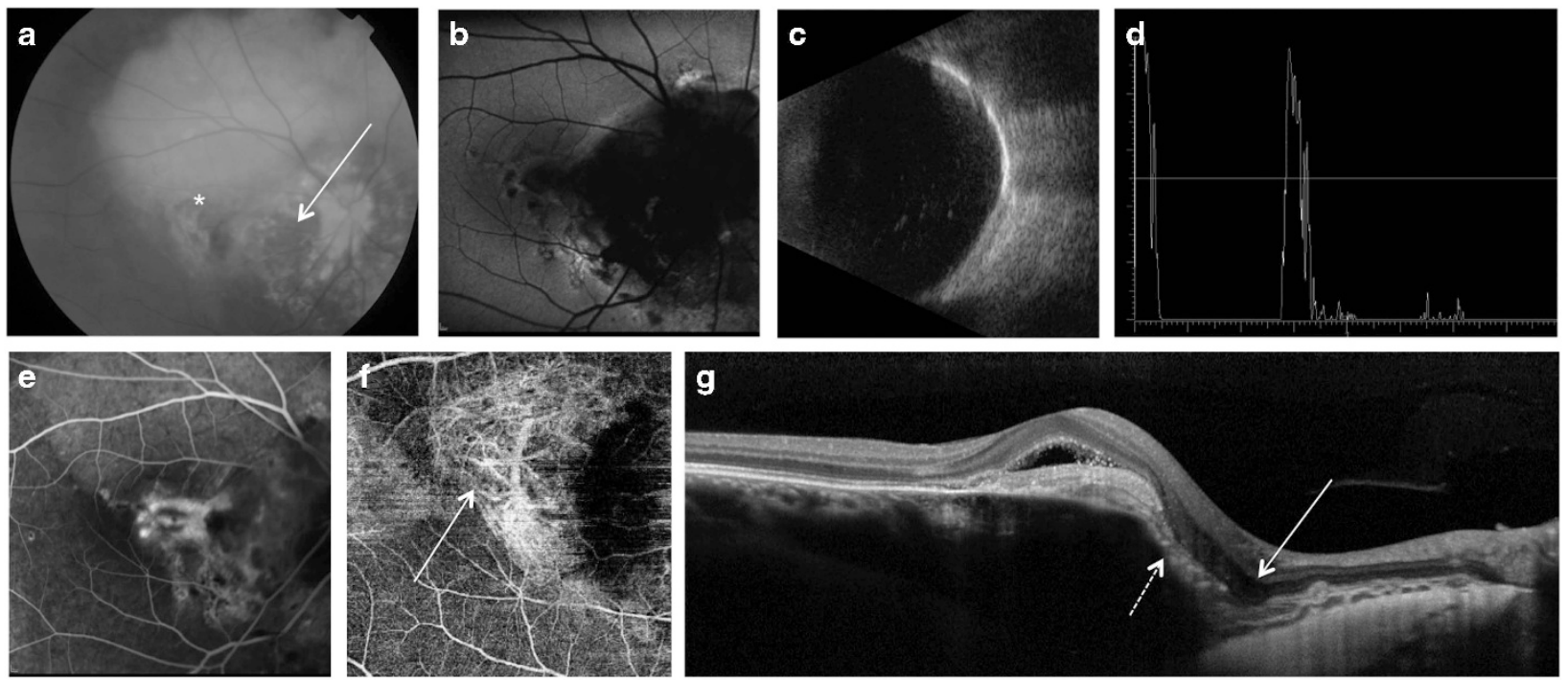

Figure 2 Multimodal imaging of right eye in case 2. Fundus photograph (a) showed a large choroidal osteoma involving the macula, with a foveal retinal hemorrhage (asterisk); a pale area within the osteoma with visibility of underlying choroidal vessels around the optic disc was suggestive of decalcification of the tumor in the peripapillary area (arrow). Blue fundus autofluorescence (FAF) (b) showed a diffuse peripapillary hypoFAF area in correspondence of the decalcified portion of the choroidal osteoma. B scan echogram (c) showed a solid mass with acoustic shadowing. A scan ultrasonography (d) showed a high reflectivity of the lesion with sound attenuation posterior to the lesion. Axial length on A scan was $24.14 \mathrm{~mm}$. Fluorescein angiography (e) showed an active leaking subfoveal CNV (arrow) which was visible on optical coherence tomography angiography (f, arrow). On optical coherence tomography scan (g) a well defined subretinal hyperreflective material was present in correspondence of the CNV; a focal choroidal excavation (arrow) was found adjacent to the CNV and in correspondence of the tumor decalcification which showed thinning of the overlying retinal pigment epithelium (dashed arrow). A full color version of this figure is available at the Eye journal online.

nonconforming lesion as a result of the progressive stress exerted on the outer retina by the initial FCE. Our case 1 was originally reported by Introini $e t a l^{10}$ who first described the progression from conforming to nonconforming FCE in a case of bilateral CO. This progression was attributed to subclinical tumor decalcification (at that time $\mathrm{CNV}$ had not occurred in the eye with FCE). ${ }^{10}$ It is noteworthy that in our case 2 a FCE was noted in correspondence of the decalcified tumor and was adjacent to the CNV (Figure 2).

Therefore it is possible that progressive decalcification of the tumor might have played a common pathogenetic role for development of CNV and FCE in these patients.

In this series, we used OCT-A which is able to image the different vascular networks of the retina and choroid in a dyeless fashion. Recent case reports have shown utility of OCT-A in detection of CNV in CO as compared to traditional contrast imaging angiography. ${ }^{11,12}$ Also in our series OCT-A showed to be a valuable tool for detection of $\mathrm{CNV}$ complicating $\mathrm{CO}$.

In summary, FCE may be associated with CNV in CO. Further studies are warranted to elucidate the relationship between FCE and CNV development in $\mathrm{CO}$.

\section{Summary}

What was known before

- Choroidal osteoma may be complicated by choroidal neovascularization (CNV).

- $\mathrm{CNV}$ in choroidal osteoma may be related to tumor decalcification.

What this study adds

- Focal choroidal excavation (FCE) may be found in eyes with choroidal osteoma and CNV.

- Decalcification of choroidal osteoma may represent a common pathogenic pathway for development of FCE and $\mathrm{CNV}$ in choroidal osteoma.

\section{Conflict of interest}

The authors declare no conflict of interest.

\section{References}

1 Shields CL, Shields JA, Augsburger JJ. Review: choroidal osteoma. Surv Ophthalmol 1988; 33: 17-27.

2 Shields CL, Sun H, Demirci H, Shields JA. Factors predictive of tumor growth, tumor decalcification, choroidal neovascularization, and visual outcome in 74 eyes with choroidal osteoma. Arch Ophthalmol 2005; 123: 1658-1666. 
3 Jampol LM, Shankle R, Tornambe P, Spaide RF, Hee MR. Diagnostic and therapeutic challenges. Retina 2006; 26: 1072-1076.

4 Margolis R, Mukkamala SK, Jampol LM, Spaide RF, Ober MD, Sorenson JA et al. The expanded spectrum of focal choroidal excavation. Arch Ophthalmol 2011; 129: 1320-1325.

5 Pierro L, Casalino G, Introini U, Gagliardi M, Sergenti J, Cascavilla ML et al. Natural course of symptomatic focal choroidal excavation. Ophthalmic Surg Lasers Imaging Retina 2015; 46: 125-130.

6 Obata R, Takahashi H, Ueta T, Yuda K, Kure K, Yanagi Y et al. Tomographic and angiographic characteristics of eyes with macular choroidal excavation. Retina 2013; 33: 1201-1210.

7 Xu H, Zeng F, Shi D, Sun X, Chen X, Bai Y. Focal choroidal excavation complicated by choroidal neovascularization. Ophthalmology 2014; 121: 246-250.
8 Kuroda Y, Tsujikawa A, Ooto S, Yamashiro K, Oishi A, Nakanishi $\mathrm{H}$ et al. Association of focal choroidal excavation with age-related macular degeneration. Invest Ophthalmol Vis Sci 2014; 55: 6046-6054.

9 Sheilds CL, Brown GC, Sharma S, Sheilds JA. Choroidal osteoma. Ryan SJ, Schachat AP. Retina. 5th edn Elsevier: London, UK: 2013; pp 2330-2339.

10 Introini U, Casalino G, Parodi MB, Bandello F, London NJ. Diagnostic and therapeutic challenges. Retina 2016; 36: 422-427.

11 Azad SV, Takkar B, Venkatesh P, Kumar A. Swept source: optical coherence tomography angiography features of choroidal osteoma with choroidal neovascular membrane. BMJ Case Rep 2016; 2016: 215899.

12 Cennamo G, Romano MR, Iovino C, Velotti N, Breve MA, de Crecchio $\mathrm{G}$ et al. OCT angiography in choroidal neovascularization secondary to choroidal osteoma. Acta Ophthalmol 2016; 95: e152-e154. 